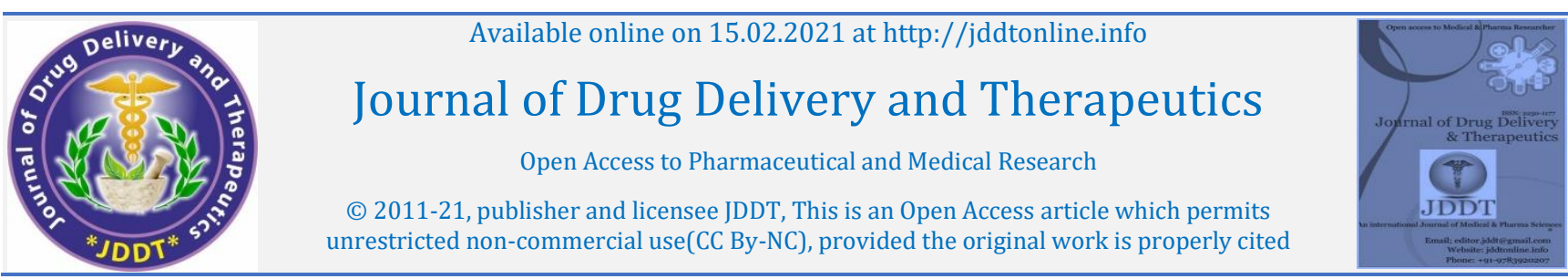

(C) 2011-21, publisher and licensee JDDT, This is an Open Access article which permits Access Full Text Article

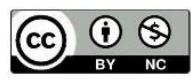

Review Article

\title{
Hydrotropic Solubilization: An Emerging Approach
}

\author{
Ashwin Kuchekar ${ }^{*}$, Ashwini Gawade ${ }^{1}$, Sanjay Boldhane ${ }^{2}$ \\ ${ }^{1}$ School of Pharmacy, Dr. Vishwanath Karad MIT World Peace University, Kothrud, Pune-411038, Maharashtra, India
}

${ }^{2}$ Micro Advanced Research Centre (MARC) No.58/3, Kudlu Village, Anekal Taluk, Singasandra Post, Bangalore, 560068, Karnataka India

Article Info:

\section{Article History:}

Received 27 Nov 2020;

Review Completed 19 Jan 2021

Accepted 28 Jan 2021;

Available online 15 Feb 2021

\section{Cite this article as:}

Kuchekar A, Gawade A, Boldhane S, Hydrotropic

Solubilization: An Emerging Approach, Journal of Drug

Delivery and Therapeutics. 2021; 11(1-s):200-206

DOI: http://dx.doi.org/10.22270/jddt.v11i1-s.4724

\section{*Address for Correspondence:}

Dr. Ashwin Kuchekar, School of Pharmacy, Dr.

Vishwanath Karad MIT World Peace University,

Kothrud, Pune-411038, Maharashtra, India
Abstract

Drug development plays an important role in patient safety and effectiveness. The therapeutic suitability of a new drug depends on the solubility. The solubility of the sparingly soluble drug remains a problem in identifying new active compounds. Solubility plays an important role in achieving optimal drug concentration. Low solubility is not only a concern for the production of formulations, but also an obstacle from the outset when identifying active chemicals for therapeutic purposes. Due to its simplicity in terms of ease of administration and economy, the oral route is the preferred route of drug administration over other routes. Effective aqueous solubility is the first prerequisite for oral medication, since low solubility has poor absorption and bioavailability and unpredictable toxicity of the gastrointestinal mucosa. To avoid these crises, different methodologies are used to improve the solubility and bioavailability of poorly soluble drugs, and hydrotropic solubilization is one of them. Hydrotropic agents have the potential to improve the solubility of water-insoluble drugs. In this review, we try to address hydrotropic solubilization methodologies.

Keywords: Hydrotropy, Micelles, Solubility, Formulation.

\section{INTRODUCTION}

Currently, a drag encountered by everyone in development of a product is the poor solubility of drug. In fact, it is getting worse day by day. Hence, determination of solubility behaviour and enhancement of solubility of drug by various approaches remains the foremost perplexing aspect. Many of the drug substance are Poorly water soluble, that hinder their claims in drug discovery. Yet the quantity of poorly soluble compounds is mounting, which causes problem for producing formulation of high bioavailability in pharmaceutical industry. 'Hydrotropic solubilization process' is the procedure used for improving the solubility by adding large amounts of solute that increases the solubility of additional solute. General terms of the solubility, determination \& various factors affecting solubility, background, chemical structure, mechanisms and applications thereof have been described in brief. Many research scientists have often encountered with the insolubility of drug substances in dosage form development. More than a third of the drugs mentioned in USP currently fall into groups of poor aqueous soluble drugs. About 41 per cent of failures in the production of new drugs are water insoluble ${ }^{1-3}$

\section{BIOPHARMACEUTICAL CLASSIFICATION SYSTEM (BCS)}

Amidon et al. (1995) commenced BCS focusing on solubility, permeability, and dissolution for predicting oral absorption of a drug. It is defining as a maximum solubility product as the maximum dose that can be soluble from $\mathrm{pH} 1$ to 7.5 at $250 \mathrm{~mL}$ or less in aqueous media. The BCS considers a substance to be highly permeable if its absorption rate in humans is 90 per cent or more of a dose given. Drugs are dependent on properties falling within one of four BCS classifications such as solubility, permeability and dissolution:

a. Class-I-Drugs having high solubility and high permeability

b. Class-II-Drug having low solubility and high permeability

c. Class-III-Drugs having high solubility and low permeability

d. Class-IV-Drugs having poor solubility and poor permeability

BCS links drug release in vitro to bioavailability in vivo, focused on drug solubility and drug permeability as essential parameters regulating drug absorption. Suggestions to set the criteria for in vitro drug dissolution and its similarity with that of the in vivo model are made based on the above listed classes of drug substance. This is a very useful method for in vitro in vivo correlations (IVIVC) that meets the requirement of a compound's physicochemical properties such as solubility and permeability. As drugs under Class I are categorized as high soluble and high permeable it becomes very difficult for achieving desirable release profile, pharmacokinetic and pharmacodynamic characteristic of dosage form. Formulation methods include both release rate regulation and other physical and chemical properties of 
drugs. The design for drugs under Class II are focused on reduction of particle size, lyophilization, and surfactant incorporation, use of complexing agents, and other novel drug delivery systems. The said class is appropriate for drug design to boost the solubility. Class III drugs require inventions which focus on specific limitations on permeability. Drugs under Class IV profess a threat in developing the drug carrier and the path of selection for the administration of drugs. More attention has to be given on formulation development side while considering class-II drugs and early attention at chemical level ${ }^{4-6}$.

\section{Determination of Solubility}

High solubility and subsequent rapid dissolution may result in a decreased bioavailability in the case of a product that is fragile in an acidic stomach condition. The solubility of new medications shall be considered as a role of $\mathrm{pH}$ over a physiological $\mathrm{pH}$ spectrum of 1 to 8 . If the solubility is labelled very low or very strong, there might be attempts to adjust it. Adjusting the $\mathrm{pH}$ values of the saturated solution becomes vital since these compounds promote their own $\mathrm{pH}$ conditions governed by respective pKa values. Literature states that others have used solutions for hydrochloric acid or sodium hydroxide $\mathrm{e}^{7-12}$.

\section{VARIOUS PARAMETERS AFFECTING ON SOLUBILITY}

\section{Effect of molecular size:}

Because of non-polar clusters \& water interactions, larger organic molecules have lower aqueous solubility. This states that solubility depends on the amount of water molecules that the solvent molecule can fit around it 13 .

\section{Effect of ionization on solubility:}

A compound's solubility at a given $\mathrm{pH}$ is a feature of the solubility of the ionized form and the neutral molecule's controlling solubility. This results in the relation between the free acid or base 's intrinsic solubility, the $\mathrm{pKa}$, and the $\mathrm{pH}$. So, the drug solubility can be determined if the intrinsic solubility at pH and pKa of the free acid or base is known ${ }^{14-17}$.

\section{Effect of salt form:}

The solution is shown to "salt in" that improve solubility and reduce to "salt out" solute. Additive effect depends on the influence on its capability to strive with solvent molecules. The effect of electrolytes on a salt's solubility is the principle of drugs which are poorly soluble. If a common ion is excessive in concentration, then additional ion should be little in a saturated substance solution. By comparison, the impact of foreign ions on the solubility of sparsely soluble salts is reverse and is so called salt effect ${ }^{18}$.

\section{Effect of temperature:}

The basic rule of dissolution is increasing the drugs solubility with increase in temperature. Hence, most temperaturedriven graphs of solubility display a continuous increase, with some exceptions ${ }^{19}$.

\section{Effect of crystal properties: Polymorphism \& related phenomenon:}

The stable crystalline form of a compound is polymorphism, which results from the possibility of at least two unlike configurations of the solid-state compound. A given compound polymorphs have diverse physico-chemical properties, like melting point, solubility \& density. Therefore, polymorphism has significant implications for formulations, biopharmaceutical \& chemical processes. There may also be solvates, hydrates \& amorphous type, in addition to polymorphs. The energy needed for a drug substance to exit from a crystal is higher than that is needed to spurt from an amorphous material. A drug substance's amorphous form is more soluble than a crystal-form. Generally, polymorphs can be divided into two different groups:

Enantiotropy: By changing the temperature or pressure, one polymorph can be reversibly transformed into another.

Monotropy: The change between the two forms is irreversible ${ }^{20-22}$.

Poor aqueous solubility primes to poor drug release and poor oral bioavailability. Boosting the solubility of drugs that have poor aqueous solubility is a challenging thing. The formation of salts, solubilization and micronisation are also used to augment product solubility, dissolution and bioavailability. Such methods have some practical limitations. Many drug candidate's water insoluble, thereby reducing their experimental applications. Such low watersolubility raises problems in the pharmaceutical industry formulating high bioavailability. Different procedures are essential for the improving the solubility of drugs which are water insoluble. There are many methods to upsurge the solubility of new molecules that are poorly soluble in water 23,24 . Present approaches to aqueous-solubility in improving the insoluble drugs are described below:
a. Reductions of particle size
b. Using co-solvents
c. Complexation of approach
d. Solid dispersion technology
e. Synthesis of prodrugs and analogues
f. Emulsions, micelles and liposomes
g. Use of carriers (natural, synthetic)
h. Use of liquid-solid techniques
i. Use of hydrotropic agents (hydrotropes)

Hydrotropy is one of those techniques where the drugs with poor aqueous solubility can be improved with the use of hydrotropic agent since it requires only the mixing of drugs with hydrotrope in water. Hydrotropy doesn't involve:
a. modification of hydrophobic substances,
b. addition of an organic solvents

Despite the advantages mentioned above, hydrotropes are not explored to improve the solubility of insoluble drugs. The main reason for this might be the employment of hydrotropic agents of low relative molecular weight leading to the coabsorption of a major amount of hydrotropic agent either from the gastrointestinal tract after oral processing. Hydrotropy means water turning to something. Hydrotropy shows rise in aqueous solubility triggered by adding significant amounts of an additional solvent. A hydrotrope is a molecule capable of increasing other molecules 'water solubility and also increasing non-ionic surfactants' solubility. Hydrotropes ability to improve organic solubility in aqueous medium is highest when hydrotropes concentration is adequate to bring the construction of micelles. To induce the formation of micelles the hydrotropes are very small. To improve the solubility of insoluble drugs, hydrotropic molecules are added at definite amounts, called as the minimum hydrotropic concentration (MHC). Variations in the solution possessions such as viscosity, conductivity, surface tension or solubility often indicate MHC. The large amounts needed to achieve MHC restrict 
hydrotropic applications. Kumar et al reported an approach to MHC reduction, by adding salts, alcohols and urea moderated the MHC of various hydrotropes. The hydrotropic consequence is perilous above a minimum hydrotrope concentration (MHC) which may be a distinctive of a specified hydrotropic agent, similar to the critical micellar concentration (CMC) of a surfactant. Since hydrotropic hydrophobic regions are small, their MHC is within the molar range. In a hydrotrope solution, the solubility of an organic compound increases strongly above the MHC, but at increased hydrotrope concentrations, it may drop based on the quality of the solute. Diluting the solution with aqueous phase to improve the dissolved solution, that differs when the hydrotropic concentration descents than its MHC as other solid or liquid phase. Hydrotropy is a process similar to, but with superior efficiency than, micellar solubilization. Amphiphilic hydrotrope compounds prefer to assemble, with hydrophobic molecules. All such aggregates are smaller than surfactant micelles, and less collective. The characteristic of hydrotropes is their ability to distinguish even strongly linked compounds between various organic constituents in a mixture. Hydrotropes have the possessions of preventing the lamellar crystal assembly of surfactants in water, producing incessant region of isotropic liquid solubility. The existence of the lamellar structure in surfactant solutions at high levels restricts the solubility of hydrophobic compounds $25-27,63,64$.

\section{BACKGROUND}

Neuberg studied the term hydrotropy by adding increased amounts of alkali metal salts of different organic acids to describe the increased solubility of the solution. He mentioned that some aqueous saline solutions have the power to dissolve certain substances that are not soluble in water. In the pharmaceutical industry, this phenomenon has been demonstrated. This was used to define non-micellar, liquid or solid, organic or inorganic substances that are capable of solubilizing insoluble compounds 28,29 .

Poochikian and Cradock hypothesized an arrangement from plane to plane, and ligand molecules coupled by electrostatic and hydrophobic interactions are essential for the solubility of insoluble compounds 30 .

Badwan et al. studied the solubility and spectral analysis which suggested a donor-recipient type electrostatic force and played a significant role in solubilization. His results showed molecular aggregation of hydrotropes at some concentrations. The concentration of aggregation increases depending on the temperature, thereby increasing the solubilizing effect. In addition, the presence of molecules was seen as the mechanism responsible for drug solubilization. It is believed that a donor-acceptor interaction between the molecules stabilizes an inclusion and determines the degree of solubility ${ }^{31}$.

Saleh and El-Khordagui recommended hydrotropy as an experience are non-related to the organic acid salts that include other cationic salts and neutral molecules ${ }^{32}$.

Winsor speculated that hydrotropy was thought to be simply another form of solubilization in targeted groups of hydrotropic agents with solute dissolved. This effect is often related to the complexing of a weak interaction between both the hydrotropic agent and the solute 33 .

Higuchi and Drubulis studied the interactions between aromatic / hydroxy aromatic compounds that maybe of lesser importance defining the form of "donor-acceptor" and the helps the hydrophobic and hydrogen bond 34 .

Seigo Ueda stated through spectrophotometric studies that solutes and sodium benzoate derivatives were interacting in aqueous solutions. The poorly water-soluble compounds used in aqueous systems have been found to form individual molecular complexes with derivatives of sodium benzoate. As an effect, it was suggested that the main factor for complexation be due to the transfer force of charge or the bond of hydrogen 35 .

Saleh and Daabis showed the impact of sodium benzoate and sodium salicylate. The spectral analysis suggested that, in addition to forming molecular complexes at low concentrations, the interaction may be due to the formation of molecular complexes and also to certain factors involved in the solubilization process ${ }^{36}$.

Hamza and Paruta studied the use UV, TLC, IR and NMR analysis in the improvement of aqueous solubility. Such experiments showed that the sodium salt hydrotropes don't have any special bond or complex formation. Analysis of the UV and TLC revealed a complexation. The additional hydrotropic agents suggested the solubilization process as "salty" by causing two formally miscible liquid phases of ternary system to be miscible ${ }^{37}$.

Feldman and Gibaldi projected that urea and methyl urea solubilize benzoic and salicylic acids by "breaking" the aqueous groups neighbouring the nonpolar part, expanding the entropy and producing a "driving" force for solubilization 38 .

Suzuki and Sunada examined the mechanism of the shift in enthalpy and proposed that intermolecular forces other than hydrogen bonds be involved in complexation ${ }^{39}$.

Jain et al. studied the hydrotropic solubilization analysis for the acquisition of the solubilization mechanism; spectral tests of solubility, spectrum, solution properties, gel formation, paste formation, TLC and IR were performed with structural variability in hydrotropes. It was due to a weak ionic interaction that the solubility increased. The formation of CMC tends to be the potential mechanism for hydrotropic solubilization at higher concentrations ${ }^{40}$.

\section{CHEMICAL STRUCTURE}

The chemical composition of the hydrotropic salts consists of two basic parts,

\section{a. presence of an anionic group}

\section{b. presence of hydrophobic aromatic ring}

The anionic group produces good water solubility that is a basic requirement for a hydrotropic substance. In contrast, hydrophobic part is a key element in the hydrotropic solubilization mechanism. Hydrotropic agents have a hydrophobic planar structure carried in by a polar group to solution. Thus, compounds with such a hydrophobic planar component and a polar group, is not inherently anionic, and behaves like hydrotropic agents 19 .

\section{Mechanisms}

The mechanism cannot describe the exact solubilization of a hydrotrope compound. Since the exact mechanisms involved in improving the aqueous solubility of compounds with hydrotropes are unknown, the functional criteria of the hydrotropes are difficult to predict solubilize a drug substance. The mechanisms that scientists have predicted are defined as follows:
a. Complex formation
b. Aggregation of hydrotrope
c. Salting - in effect
d. Water structure changes
e. Combination of above mechanisms 
Table 1: shows the hydrotropic solubilization of numerous poor aqueous soluble compounds with their respective mechanisms.

\begin{tabular}{|c|c|c|c|c|}
\hline $\begin{array}{l}\text { Sr. } \\
\text { No. }\end{array}$ & Hydrotropic substance & Drug & Proposed mechanism & References \\
\hline \multirow{5}{*}{1} & \multirow{5}{*}{ Sodium salicylate } & Temazepam & Complex formation & 53 \\
\hline & & Diazepam & Electron- donor or & \multirow{2}{*}{31} \\
\hline & & Clonazepam & Electron- receptor reaction & \\
\hline & & Acetohexamide & Salting - in & 53 \\
\hline & & Nifidipine & Complex formation & 39 \\
\hline \multirow{3}{*}{2} & \multirow{3}{*}{ Nicotinamide } & Riboflavin & Self- association & 55 \\
\hline & & Chlorthiazide & Complex formation & 56 \\
\hline & & Ketoprofen & Aggregation & 48 \\
\hline \multirow{3}{*}{3} & \multirow{3}{*}{ Sodium Benzoate } & Flubiprofen & Molecular aggregation & 54 \\
\hline & & Ketoprofen & Aggregation & 57 \\
\hline & & Phenacetine & Complex formation & 58 \\
\hline 4 & Sodium gentisate & Paracetamol & Complex formation & 37 \\
\hline 5 & Sodium glycinate & Paracetamol & No complex & 37 \\
\hline 6 & $\beta$-cyclodextrin & Clotrimazole & Complex formation & 59 \\
\hline 7 & Urea & Acetazolamide & Complex formation & 60 \\
\hline 8 & Sodium toluate & Allopurinol & Complex formation & 53 \\
\hline 9 & Sodium Ibuprofen & Ibuprofen & Aggregation & 61 \\
\hline 10 & Gentisic acid & Acetazolamide & Complex formation & 60 \\
\hline 11 & Isoniazide & Thioacetone & Self-association & 52 \\
\hline
\end{tabular}

\section{Complex Formation:}

Complexation is by far a widely suggested explanation for understanding the higher solubility of the compound resultant from the addition of hydrotrope. It can be defined as a reversible combination of substrate molecules with molecules of ligand species to form a new species with constant association. One of the physical properties used for hydrotropic behaviour is to increase the solubility of the complexation. Complexation characterisation methods include IR spectroscopy, UV spectroscopy, NMR, X-ray crystallography, etc.

\section{Self-association or aggregation:}

Evidence of aggregation formation was predicted from measurements of surface tension, specific conductivity, and viscosity. Hydrotrope aggregation has been proposed as a mechanism to explain solubility. The minimum critical hydrotropic concentration (MHC) of the hydrotrope on the phase solubility diagram correlates with the surface tension measurements. The critical concentration of certain hydrotropes cannot be determined in certain materials due to the gradual self-association process. Due to this staged process, there is no inflection point due to surface tension as the amount of solute in the solution increases. Therefore, the method of using the minimum hydrotropic concentration cannot be applied as a universal tool for hydrotropic behaviour.

\section{Salting - In effect:}

This approach came from the addition of electrolytes to a non-electrolyte solution where the non-electrolyte can either increase the solubility or precipitate out. The former is called the salting-in effect and the later the salting-out effect.

\section{Water structure change:}

It is reported that alteration of water structure occurs by addition of hydrotropic agent. The $-\mathrm{OH}$ group forms a hydrogen bond with a hydrotrope while - $\mathrm{NH} 2$ is not able to form the hydrogen bond with any of the hydrotrope. This aggregate of hydrotrope may induce an increased structuring and hydrophobicity of the water. This contributes to change in water structure. Urea and nicotinamide were descried as the water breaker. Nicotinamide acting as water structure breaker may decrease the hydrogen-hydrogen bonding in water, so this effect may be the reason for increase solubility of drugs.

\section{Solvent structure:}

To understand the interaction of hydrotrope with solvent system, one has to be perfect with solvent structure. The structure of solvent systems can be altered by additives. Marcus introduced the concept of structuredness of solvents in better understanding the structure of solvents. Stiffness, openness and ordering are the basic characteristics of solvents. 
Stiffness- Considered by the amount of work required to create a solvent cavity

Openness- Property referring to free solvent volume which in turn related to fluidity

Ordering- Property relating to entropy deficit 39,42 .

\section{APPLICATIONS}

\section{Solid dispersion}

Solid dispersions in aqueous phase enhance the drug release rate and bioavailability of drugs. Popular methods used to prepare solid dispersions include evaporation of the solvent, fusion and oxidation of the solvents. In the solvent evaporation process, an organic solvent is used for the dissolution of both the substance and the system. A proper evaporation procedure obtains a strong dispersion. The principal disadvantages of this method are residual solvent toxicity, higher solvent cost and contamination. The main feature of this method is that the hydrotropic agent is water soluble whilst the drug is water insoluble. But the product dissolves in the presence of a significant quantity of hydrotropic agent in water. Using an evaporation technique, the water is then removed to obtain a solid mass (solid dispersion). Water is not a solvent for drugs with poor aqueous solubility in the absence of a hydrotropic agent; therefore the proposed method differs from the existing solvent approach and represents a new application of the hydrotropic solubilization phenomenon. Urea, used to make solid dispersions of a drugs with poor aqueous solubility. The simplicity of the technique and the ease of large-scale manufacturing make hydrotropic solid dispersions commercially profitable by helping to boost the therapeutic potential of their poorly water soluble solid oral drug forms. The associated problems are a delayed onset of action and can be solved by the effect of their solid dispersions using a hydrotropical solubilization technique to achieve the maximum plasma concentration of poorly water-soluble drugs. Hydrotropic method is cost-effective, environmentally friendly and safe in contrast with the method of solvent evaporation allowing the use of organic solvent. This method formulates solid dispersions of drugs with poor aqueous solubility for an increased drug release and, thus, bioavailability. The formulations developed are less expensive than the commercial formulations which use expensive additives / excipients 43 .

\section{Mixed Hydrotropy}

There is remarkable synergistic effect of combining two hydrotropic molecules on increasing the solubility of drugs with poor aqueous solubility. In spectrophotometric analysis of drugs which are poorly soluble in water, R.K. Maheshwari has developed mixed hydrotropy. In a 45 per cent urea solution and a 45 per cent sodium citrate solution (SCS), the increase in Aceclofenac solubility was more than 20 and 40 times greater relative to its solubility in aqueous phase. Enhancing Aceclofenac solubility in a mixed hydrotropic solution containing 22.5 per cent urea and 22.5 per cent sodium citrate has been more than 700 time. The mixed hydrotropic solution technique is used to dissolve poorly soluble in drugs in aqueous phase. The mixed hydrotropic solution method is used to dissolve poorly water-soluble substances. For the spectrophotometric study of products poorly soluble in water, most organic solvents such as ethanol, methanol, acetonitrile, hexane, cyclohexane, diethyl ether, chloroform and toluene are used. Some of these organic solvents are potentially toxic and more expensive. A disadvantage of organic solvents is the accuracy of the spectrophotometric calculation due to volatility. Because urea and sodium citrate do not interfere above $245 \mathrm{~nm}$, it is possible to assess poorly water-soluble drugs above $245 \mathrm{~nm}$ using mixed hydrotropy, and evading the usage of organic solvents. This method is novel, quick, cost effective, safe, precise, accurate and environmentally friendly. Several other hydrotropes are also sought by linking them to utilize stimulatory activity on drugs with poor aqueous solubility. In addition, mixed hydrotropy will be used in designing aqueous formulations of drugs with poor aqueous solubility $33,44,45$.

\section{Liquid oral solutions (syrup)}

The industrial pharmacist faces many challenges in formulating and designing solutions. Special techniques are needed to dissolve drugs that are poorly soluble in water. A medicinal substance in solution is ready for absorption instantly and, is absorbed faster and more proficiently. The marketable poorly water-soluble oral liquid dosage types of drugs are mainly in the form of suspensions. Liquid oral solutions (syrups) have greater bioavailability compared to suspensions and a quicker onset of action. Urea is non-toxic in nature. Other poorly water-soluble drugs are given opportunities to develop liquid solutions as syrups using appropriate hydrotropic agents. Thus, if the chemical equilibrium remains unchanged, solutions are better established than suspensions. Dosage types of a solution form show greater physical stability than suspensions. Suspensions have their own instability, such as developing cake, and growing crystals. The proposed techniques are simple, practical, effective and less costly than the commercialized formulations using costly additives / excipients. Hydrotropic agents are safe and suggest adoptability for large-scale production. Metronidazole, a drug with poor aqueous solubility, was selected for the formulation of a liquid oral solution (syrup) using urea, as a hydrotropic agent to give a rapid effect and better bioavailability 46 .

\section{Quantitative analysis}

Quantitative study of drugs which have poor aqueous solubility entails different organic solvents. Sodium benzoate is among the different hydrotropic agents widely used, and other drugs with poorly aqueous solubility denote enhanced solubility. Such drugs are tested successfully in solid dosage forms. Recovery experiments and statistical evidence proved the precision, reproducibility, and consistency of the techniques suggested. It is found that concentrated hydrotropic aqueous solutions of sodium benzoate, sodium salicylate, urea, nicotinamide, sodium citrate and sodium acetate increase the water solubility of drugs. Maheshwari et al employed sodium benzoate as a hydrotropic agent to quantify drugs with poor aqueous solubility like loxacin, norfloxacin, nalidixic acid, metronidazole, and tinidazole drugs by spectrophotometric analysis and titrimetric aspirin analysis. Among three drugs, those have poor aqueous solubility, ibuprofen, flurbiprofen and naproxen, a $2 \mathrm{M}$ sodium benzoate solution was used as a solubilizing agent. In a $2 \mathrm{M}$ sodium benzoate solution, the solubilities of ibuprofen, flurbiprofen and naproxen increased by more than 80,110 and 120 times compared with the solubilities in distilled water, respectively. In its titrimetric tests, a $2 \mathrm{M}$ sodium benzoate solution was used to disperse these products. Methanol, ethanol, chloroform, dimethylformamide, and acetone are different organic solvents used to dissolve drugs with poor aqueous solubility allowing acid-base titrations. High cost, instability and toxicity are the main disadvantages of organic solvents $23,24,47,48$. 


\section{Parenteral preparations}

Acquired immunodeficiency syndrome (AIDS) remains one of the most important challenges facing the world today. Due to their low bioavailability, it has been shown that the formulations currently available for this disease are less effective and lead to low efficiency. N. K. Jain and M Nahar have developed an aqueous injection of Saquinavir that would be much more effective, safer and with fewer side effects. They studied the solubilization of saquinavir, which is almost Poorly water soluble due to the addition of hydrotropes and cosolvents, showed an improvement in the solubility of saquinavir. This may be due to weak interactions of ions at low concentrations. At high concentrations, it is likely to evolve a mechanism of complexation and self-aggregation 6,25-27.

\section{Hydrotropic nanocarriers}

Hydrotropic agents such as dendrimers and polymeric micelles in the form of nanocarriers improve the solubility in water. For poorly water-soluble drugs, hydrotropic polymers, dendrimers and micelles are formed as nanocarriers. Paclitaxel a hydrophobic drug used to reveal the solubility efficacy improving characteristics of hydrotropic nanocarriers. Hydrotropic polymers and dendrimers have benefits for paclitaxel solubilization in terms of rising the local hydrotropic concentration in aqueous media. The main advantages of hydrotropic polymeric micelles over traditional polymeric micelles are that the interaction between the polymer segment and paclitaxel is based on the miscibility between the two rather than the hydrophobic interaction alone. Hydrotropic polymeric micelles are much more stable in aqueous solution as compared to traditional polymeric micelles. Hydrotropic dendrimer micelles were developed by self-assembly dendrimer conjugates for cholesterol. The dendrimer represented as self-assembly might dissolve both the paclitaxel and the dendrimer. New systems based on hydrotropical and dendrimer polymers offer a new method to the design of low solubility nanocarriers for drugs. For their elevated efficiency in drug loading and long-term stability, hydrotropic nanocarriers have benefits 49 .

\section{Industrial applications}

The use of hydrotropes is particularly beneficial in industrial applications due to the rapid retrieval of solutes in the solution. At the same time, hydrotrope solutions do not respond to an emulsification problem that generally occurs with traditional surfactant solutions. For example, Natoluene sulfonate, a hydrotrope molecule, has a strong polar ionic group and smaller hydrocarbon than traditional surfactant molecule. Therefore, due to their amphiphilic structural properties, hydrotropes and surfactants are used in aqueous solutions 50 .

\section{Controlled Release preparations}

Hydrotropes' capacity to change the solubility of certain compounds in a substrate can be employed for entrapment and drug delivery. PTX (paclitaxel) can be dissolved in the hydrophobic centre of pol (ethylene glycol) -polymer (phenylalanine) copolymer micelles in aqueous phase; however, it is slow to extract PTX in water phase. Adding molar sodium salicylate to the micellar solution improves PTX's aqueous solubility by a factor of 100 , without altering the polymer micelles, leading to controlled release of bulk PTX. Triclosan (TCN), antimicrobial agent has slightly water solubility is entrapped in non-ionic surfactant micelles for operative administration. Apparently, TCN is inactivated in micelles in insoluble core and to be released in the water to be effective. Adding hydrotropes to the surfactant / TCN solution interferes with the micelles and helps in releasing TCN. The hydrotrope also improves the solubility of TCN, thus avoiding perception $51,52,65,66$.

\section{CONCLUSION}

In the development of formulations, the use of hydrotropic agents is widely known. Several laboratory studies have verified both its solubility and its non-flammable and nontoxic environmental quality. However, there are several questions about its mechanism based on the structure and toxicity profile, as its critical adverse effects on normal cells have not yet been tested. Competitive developments in hydrotropy and new approaches to drug delivery will bring practices closer to hydrotropic processes, stability of biological solutions, biocompatibility, and best performance and management strategies. This technique should transform progress toward improving the therapeutic contribution of poorly water-soluble drugs.

\section{ACKNOWLEDGEMENT}

The authors express special thanks to Dr. Bhanudas Kuchekar (Dean, School of Pharmacy, Dr. Vishwanath Karad MIT World Peace University, Kothrud, Pune-411038, Maharashtra, India) for their kind support.

\section{CONFLICTS OF INTEREST}

The authors declare that there is no conflict of interests regarding the publication of this manuscript.

\section{REFERENCES}

1. Rong, L. Water insoluble drug formulation, Second ed., CRS Press, Taylor and Francis Group,2008

2. Martin, S. and Karl, B., Chasing equilibrium: measuring the intrinsic solubility of weak acids and bases. Anal. Chem. 2005; 77(4):983-990.

3. Horter, D., Dressman, J. B. Influence of physicochemical properties on dissolution of drugs in the gastrointestinal tract. Adv Drug Deliv Rev. 2001; 46(1-3):75-87.

4. Devane, J., Oral drug delivery technology: Addressing the solubility/permeability paradigm. Pharm. Technol. 1998; (11):68-74.

5. Asmat, M., Syed, N. R. and Nisar, A. K. Hydrotrophy: Novel solubility enhancement technique: a review. IJPSR, 2019; 10(3):1025-1036.

6. Thipsay, M.P., Ross, S., Zhang, F., Bandari, S., Repka, M.A. Preparation of a crystalline salt of indomethacin and tromethamine by hot melt extrusion technology. Eur J Pharm Biopharm. 2018; 131(10):109-119.

7. Khadka, P. Et al. Pharmaceutical particle technologies: an approach to improve drug solubility, dissolution and bioavailability. Asian J. Pharm. Sci. (2014; 9:304-316.

8. Brahmankar, D., Jaiswal, S., Biopharmaceutics and Pharmacokinetics: A Treatise. Third ed., Vallabh Prakashan, India, 2011.

9. Vemula, V., Lagishetty, V., Lingala, S. Solubility enhancement techniques. Int. J. Pharm. Sci. Rev. Res. 2010; (5):41-51.

10. Serajuddin, T. M., Mufson, D., pH-Solubility profiles of organic bases and their hydrochloride salts. Pharm Res. 1985; ( 5): =6568.

11. Kevin W. G., Richard A. P. Aqueous solubility properties of a dibasic peptide-like compound. International Journal of Pharmaceutics. 1990; 63(2): =15:167-172.

12. Serajuddin, T. M., Jarowski, C. I. Effect of diffusion layer $\mathrm{pH}$ and solubility on the dissolution rate of pharmaceutical bases and their hydrochloride salts I: Phenazopyridine. J. Pharm. Sci. 1985; (74):142.

13. Hodgdon, T., Kaler, E., Hydrotropic solutions. Curr. Opin. Colloid. In. 2007; 12:121-128.

14. Saleh, L., Khordagui, E. Hydrotropic agents: A new definition. Int J. Pharm. 1985; 24:231-238.

15. Tooru, O., Sang, C. L., Kang, M. H., Kinam, P., Mozafari, M. hydrotropic nanocarriers for poorly soluble drugs. Nanocarrier Technologies: Frontiers of Nanotherapy. 2006; 51-73. 
16. Girija, R., Vilas, G. G. Extraction of piperine from piper nigrum (black pepper) by hydrotropic solubilization. Ind. Eng. Chem. Res. 2002; (41):2966-2976.

17. Jain, N., Patel, V., Taneja, L., Hydrotropic solubilization of nifedipine. Pharmazie. 1988; (43):194-196.

18. Kumar, V., Raja, C., Jayakumar, C. A review on solubility enhancement using hydrotropic phenomena. Int. J. Pharm. Pharm. Sci. 2014; (6):1-7.

19. Dhapte V, Mehta, P. Advances in hydrotropic solutions: An updated review, St. Petersburg Polytechnical University Journal: Physics and Mathematics. 2015; (1): 424-435.

20. Maheshwari, R.K. et al. Novel application of hydrotropic solubilizing additives in the estimation of aspirin in tablets. Indian. J. Pharm. Sci. 2010; (72): 649-651.

21. Maheshwari, R. K. Mixed hydrotropy in spectrophotometric analysis of Aceclofenac. The Indian Pharmacist. 2007; 64(6):6769.

22. Maheshwari, R.K., Indurkhya, A. Formulation and evaluation of aceclofenac injection made by mixed hydrotropic solubilization technique. Iran. J. Pharm. Res. 2010; 9:233-242.

23. Maheshwari, R. K., Chaturvedi S. C., Jain, N.K. Novel application of hydrotropic solubilization in the quantitative analysis of some NSAIDs and their solid dosage forms. Ind. J. Pharm. Sci.2007; 69(1):101-105.

24. Ravi, S. S., Asha, P., Soni, M. L., Modi, V., Jaliwala. Y. A Quantitative spectrophotometric estimation of Cefadroxil using hydrotropic solubilization technique. Asian J Pharm. 2008; 7:146- 147.

25. Jain, K. Solubilization of indomethacin using hydrotropes for aqueous injection. Eur. J Pharm. Biopharm. 2007; (68):701-714.

26. Nahar, M., Jain, N. K. Formulation and evaluation of Sanquir injection. Ind. J Pharm. Sci. 2006; 10:608- 614

27. Agrawal, S., Pancholi, S.S., Jain, N.K., Agrawal, G.P. Hydrotropic solubilization of nimesulide for parenteral administration. Int. J Pharm. 2004; (274): 149-155.

28. Neuberg, C. Hydrotropic phenomena. Biochem. Z. 1916; 76:107108.

29. Neuberg, C. Hydrotrophy. Biochem. J. Pharm., 1989; 75(7):577.

30. Poochikian, G.D., Gradock, J.C., Enhanced chartreusin solubility by hydroxybenzoate hydrotropy. J.Pharm. Sci. 1979; (68):728732.

31. Badwan, A.A., Khordagui E., Saleh, A.M. and Khalil, S.A., The solubility of benzodiazepines in sodium salicylate solution and a proposed mechanism of hydrotropic solubilization. Int. J. Pharm. 1983; 13:67-74.

32. Saleh, A.M. and Khordagui E., Hydrotropic agents: a new definition. Int. J. Pharm. 1985; 24:231.

33. Winsor, P.A., Hydrotropy, Solubilization and related emulsification process. Trans Faraday Soc., 1950; 54:762-772.

34. Higuchi, T., Drubulis, A., Complexation of organic substance in aqueous solution by hydroxy aromatic acids and their salts relative contribution of several factors to the overall effects. J Pharm. Sci. 1961; 50:905.

35. Ueda S. The mechanisms of solubilization of water insoluble substances with sodium benzoate derivatives-I. Chem Pharm Bull. 1966; (14): 22.

36. Saleh, A. M., Daabis, N. A. Study of the Interaction of Menadione with Hydrotropic Salts Pharmazie. 1974; 29(8):525-7

37. Hamza, Y.E., Paruta, A.N., Enhanced solubility of paracetamol by various hydrotropic agents. Drug Dev. Ind. Pharm. 1985; (11):1577.

38. Feldman, S., Gibaldi, M. Effect of urea on solubility, role of water structure. M., J. Pharm. Sci. 1967; (56):370.

39. Suzuki, H., Sunada, H. mechanistic studies on hydrotropic solubilization of nifedipine in nicotinamide solution. Chem Pharm Bull. 1998; 46(1):125-30.

40. Jain, N.K., Agrawal, R.K., Singhai, A.K. Formulation of aqueous injection of carbamazepine. Pharmazie. 1990; (45):221-222.

41. Agarwal, M., Gaikar,V. G. Extractive separations using hydrotropes, Sep. Technol. 1992; (2):79-84

42. Rathore, K. S., Tanwar, Y. S., Nema, R. K., Gupta, G. D. A review on hydrotropes; compounds for solubility enhancement of poorly water-soluble compounds. The Pharma Review. 2007; (12).

43. Maheshwari, R. K., Indurkhya, A. Novel application of mixed hydrotropic solubilization technique in the formulation and evaluation of hydrotropic solid dispersion of aceclofenac, Asian J. Pharm 2010; 4:235-238

44. Madan, J., Pawar, K., Dua, K. Solubility enhancement studies on lurasidone hydrochloride using mixed hydrotropy. Int. J. Pharm. Investig. 2015; 5:114-120.

45. Ruchi, J. et al. Novel UV spectrophotometer methods for quantitative estimation of metronidazole and furazolidone using mixed hydrotropy solubilization. Arabian Journal of Chemistry. 2017; 10:151-156.

46. Lai K, Liquid Detergents, second ed., CRC Press, Boca Raton, FL, 2006

47. Ansari K, Gaikar V, Green hydrotropic extraction technology for delignification of sugarcane bagasse by using alky benzene sulfonates as hydrotropes. Chem. Eng. Sci. 2014; 115:11571166

48. Gaikar, V., Sharma, M. Separations with hydrotropes, Sep. Technol. 1993; 3:2-11.

49. Kumar, V., Raja, C., Jayakumar, C., A review on solubility enhancement using hydrotropic phenomena. Int. J. Pharm. Pharm. Sci. 2014; 6:1-7.

50. Rub, M., Azum, N., Kumar, D., Khan, F., Asiri, A. Clouding phenomenon of amphiphilic drug promazine hydrochloride solutions: Influence of pharmaceutical excipients., J. Ind. Eng. Chem. 2015; 21:1119-1126.

51. Kamble, S., Kumbhar, A., Jadhav, S., Salunkhe, R., Micheal, A Reaction in glycerol as a sustainable hydrotropic medium. Mater. Today: Proc. 2015; 5:1792-1798.

52. Jyoti, J., Nidhi, N. S., Vikas, A. S., A review on hydrotropy: a potential approach for the solubility enhancement of poorly soluble drug. Asian J Pharm Clin Res, 2019; 12(10):19-26.

53. Ammar, H.O., Nahhas, Effect of aromatic hydrotropes on the solubility of allopurinol Part 3: Sodium Salts of Toluic acids, Pharmazie. 1993; 48(H10):751-754

54. Khalafallah, N., Hammouda, Y. The solubility and Complexing properties of acetohexamide in the presence of hydrotropic agents. Pharmazie. 1973; 28(H7):452-454.

55. Coffman, R.E., Kildsig D.O. Self Association of Nicotinamide in aqueous solution: Light scattering and Vapour pressure Osmometry studies. J. Pharm. Sci. 1996; 85(8):848-853.

56. Ammar, H.O., Ibrahim, S.A., El-Faham, T. H. Interaction of chlorothiazide and hydrochlorothiazide with certain amide, Imides and xanthaines. Pharm. Ind. 1981; 43(Nr 3):292-295.

57. Jain, N. K., Singhai, A. K., Jain, S. Hydrotropic solubilization of ketoprofen. Pharmazie, 1996; 51(4):236-239.

58. Ammar, H.O., Nahhas, Effect of aromatic hydrotropes on the solubility of allopurinol Part 2: Effect of nicotinamide and sodium salts of benzoic, napthoic and nicotinic acid Pharmazie. 1993; 48(H7):534-536

59. Pedersen, M., Effect of hydrotropic substances on the complexation of clotrimazole with $\beta$-cyclodextrin. Drug dev. Ind. Pharm. 1993; 19(4):439-448.

60. Elsamaligy, M.S., Hamza, Y.E., Abd-Elgawad, N. A. Hydrotropic and complexing solubilization of acetazolamide. Pharm. Ind. 1992; 54(Nr5):474-477.

61. Gaikar, V. G., Latha, V. Hydrotropic properties of sodium salts of ibuprofen. Drug Del. Ind. Pharm. 1997; 23(3):309-312.

62. Zughul, M. B., Omari, M., Badwan, A. A. Enhancement of thioacetone solubility by Isoniazide in aqueous solutions. Drug Dev. Ind. Pharm. 1996; 22(9 and 10):1013-1019.

63. Kavita, S., Ashu, S., Singha, S. K., Kakkar, S. Self-Emulsifying Drug Delivery System: A Tool in Solubility Enhancement of Poorly Soluble Drugs. Indo Global Journal of Pharmaceutical Sciences, 2012; 2(3):313-332

64. Kumari, R., Chandel, P. A Kapoor. Paramount Role of Solid Dispersion in Enhancement of Solubility. Indo Global Journal of Pharmaceutical Sciences, 2013; 3(1):78-89.

65. Gawade, A. R., Boldhane, S. P. Solid Dosage Form Development of Dabigatran Etexilate Mesylate With Increased Solubility and Dissolution Using Cocrystallization. IJPSR, 2020; 11(5):28992905.

66. Kuchekar, A. B., \& Pawar, A. P. Capecitabine loaded polymeric micelles: Formulation, characterization and cytotoxicity study. International Conference on Advanced Nanomaterials \& Emerging Engineering Technologies. 2013, pp. 412-415. 